\title{
Biomarkers of Exposure to Chemical Contamination in the Commercial Fish Species Lepidopus caudatus (Euphrasen, 1788): A Particular Focus on Plastic Additives
}

OPEN ACCESS

Edited by:

Rubina Sirri,

University of Bologna, Italy

Reviewed by:

Annalisa Zaccaroni,

University of Bologna, Italy

Milad Adel,

Iranian Fisheries Research

Organization, Iran

*Correspondence:

Maria Violetta Brundo

mvbrundo@unict.it

orcid.org/0000-0001-5146-3564

Specialty section:

This article was submitted to

Aquatic Physiology,

a section of the journal

Frontiers in Physiology

Received: 11 January 2019 Accepted: 01 July 2019

Published: 16 July 2019

Citation:

Salvaggio A, Tiralongo $F$,

Krasakopoulou E, Marmara D,

Giovos I, Crupi R, Messina G,

Lombardo BM, Marzullo A,

Pecoraro R, Scalisi EM, Copat C,

Zuccarello P, Ferrante $M$ and

Brundo MV (2019) Biomarkers

of Exposure to Chemical

Contamination in the Commercial Fish

Species Lepidopus caudatus

(Euphrasen, 1788): A Particular Focus

on Plastic Additives.

Front. Physiol. 10:905.

doi: 10.3389/fphys.2019.00905

\begin{abstract}
Antonio Salvaggio ${ }^{1}$, Francesco Tiralongo ${ }^{2}$, Evangelia Krasakopoulou ${ }^{3}$, Dimitra Marmara ${ }^{3}$, Ioannis Giovos ${ }^{4}$, Rosalia Crupi ${ }^{5}$, Giuseppina Messina ${ }^{2}$, Bianca Maria Lombardo², Alessandra Marzullo², Roberta Pecoraro², Elena Maria Scalisi ${ }^{2}$, Chiara Copat ${ }^{6}$, Pietro Zuccarello ${ }^{6}$, Margherita Ferrante ${ }^{6}$ and Maria Violetta Brundo ${ }^{2 *}$
\end{abstract}

' Experimental Zooprophylactic Institute of Sicily A. Mirri, Palermo, Italy, ${ }^{2}$ Department of Biological, Geological and Environmental Sciences, University of Catania, Catania, Italy, ${ }^{3}$ Department of Marine Sciences, University of the Aegean, Mytilene, Greece, ${ }^{4}$ iSEA, Environmental Organization for the Preservation of the Aquatic Ecosystems Ochi Av., Thessaloniki, Greece, ${ }^{5}$ Department of Chemical, Biological, Pharmaceutical and Environmental Sciences, University of Messina, Messina, Italy, ${ }^{6}$ Department of Medical, Surgery Sciences and Advanced Technologies, G. F. Ingrassia, University of Catania, Catania, Italy

In recent years, the Mediterranean Sea has become an accumulation zone for waste generated by the 22 countries bordering its shores. Although the effects of plastic litter on the marine environment and on organisms have recently been studied in other areas, further information is needed for the Mediterranean Sea and, in particular, about plastics additives inputs and interactions with the biota and the trophic network, such as phthalates and bisphenol A. Plastic material production, use and disposal contribute also to the release of heavy metals into the environment, such as mercury $(\mathrm{Hg})$, often used during the production of chlorine, the primary ingredient in PVC, lead $(\mathrm{Pb})$ and cadmium (Cd), which are used as stabilizers in PVC and leach out of products during use and disposal. Our research aims to evaluate phthalates, bisphenol A and heavy metals contamination in Lepidopus caudatus (Pisces, Trichiuridae), which could be considered as a potential sentinel species. For the evaluation of toxicological effects, we evaluated the expression of vitellogenin and metallothioneins 1. In all samples analyzed, we have not found microplastics in the gastrointestinal tract but chemical analysis revealed the presence of high content of phthalates, and in particular high quantities of DIDP, DEHP, bis-benzylester phthalate, bis-butyl ester phthalate and mono-N-butyl ester phthalate in different organs. Instead, trace elements detected in tissue revealed a trend of concentrations generally higher in liver and intestine than gill and muscle tissues. Immunohistochemical analysis for anti-metallothionein 1 antibody showed a strong positivity of liver cells, both in females and males. Analysis for the anti-vitellogenin antibody showed in females a strong positivity both in the liver cells and in the gonads, in male specimens was found to be always negative except for a specimen, in which it was highlighted a positivity in some areas of the liver and of the gonad.

Keywords: fish, phthalates, bisphenol A, heavy metals, biomarkers 


\section{INTRODUCTION}

Marine pollution is one of the biggest threats on a global scale to the heart health. In the last few decades, bioaccumulation studies performed with a multimarkers approach have been a valuable tool for the investigation of environmental and animal safety (Ferrante et al., 2017). Together with the climate change, plasticizers represent an emerging problem that could influence the human ability to preserve biological diversity in the future (Jambeck et al., 2015). It is estimated that about eight million tons of plastic reach the marine environment each year (Jambeck et al., 2015). The Mediterranean Sea, a crucial hotspot for biodiversity, has been described as one of the areas most affected by marine litter in the world (Cozar et al., 2015; UNEP, 2016). Plastic accounts for 95\% of the offshore waste, on the seabed and on the beaches of the Mediterranean Sea, and comes mainly from Turkey and Spain, followed by Italy, Egypt and France (Alessi et al., 2018). Therefore, the dramatic increase in the use of plastic materials in the last decades has led to the dispersion of plasticizers in the marine environment (Zuccarello et al., 2018).

Recently, awareness is growing about how the smaller plastic fragments, the so-called microplastics (fragments less than $5 \mathrm{~mm}$ ), are harmful and dangerous to the environment and human health (Zuccarello et al., 2019). Their impact on the marine ecosystem is still under investigation, although some important implications are already known, such as the possibility to be ingested by a wide variety of marine organisms and the consequent introduction into trophic cycle (Ashton et al., 2010; Fossi et al., 2012; Seltenrich, 2015) with deleterious effects on health of marine organisms (e.g., abrasions, blockage of the digestive tract, absorption of harmful compounds) (Rios et al., 2007; Teuten et al., 2009; Engler, 2012). Plastic additives, such as phthalates and bisphenol A (BPA), are added to plastics during production processes in order to improve their properties. Since they are not covalently bound but simply mixed with the plastic polymer, they disperse easily in the environment, especially when plastic products are degraded into microplastics (Hermabessiere et al., 2017; Savoca et al., 2018). Also, many heavy metals such as $\mathrm{Cd}, \mathrm{Pb}, \mathrm{Sb}$ and $\mathrm{Sn}$ (as organotin) have been used as plastic additives, and inappropriate plastic use, disposal and recycling may lead to their undesirable release (Hahladakis et al., 2018).

In addition, due to their hydrophobic nature and to the large surface/volume ratio, microplastics are can absorb a series of toxic from seawater, bioaccumulative and persistent substances, such as polycyclic aromatic hydrocarbons (PAHs), polychlorinated biphenyls (PCBs), other persistent organic pollutants (POPs) and heavy metals (Teuten et al., 2009; Rochman et al., 2014). Single and combined effects of microplastics and other contaminants were studied in some marine organisms (Davarpanah and Guilhermino, 2015; Karami et al., 2016).

These chemicals have the potential to accumulate in the tissues of marine organisms and cause specific effects, including behavioral changes, changes in metabolic processes and endocrine disruption (Anderson et al., 2016). The endocrine system performs fundamental tasks for the life of an organism and the hormones produced by the endocrine glands have the task of controlling delicate and complex phenomena such as reproduction, growth, development, as well as the metabolism. Endocrine disrupters (EDCs) are able to imitate, compete or stop the synthesis of endogenous hormones (Avio et al., 2015, 2017); this translates into alterations of glands' function, alteration and reduction of reproduction with consequent low birth rates and potential loss of biodiversity (Gore et al., 2015). Compounds identified as EDCs, such as phthalates, bisphenol $\mathrm{A}$ and heavy metals, are a major concern for marine organisms. Low-level exposure of these substances leads to both transient and permanent changes in the endocrine system (Greenwell, 2014; Zuccarello et al., 2018). Phthalates and bisphenol A, showed to have a role in the development of obesity and glucose metabolism disorders (Stojanoska et al., 2017). Exposure to phthalates, particularly to DEHP, causes a decrease in the production of testicular testosterone in rodents, and most of the reproductive toxic effects are suggested to be related to their antiandrogenic potential (Jones et al., 1993; Erkekoglu et al., 2011). These substances were shown to cause both hepatocellular carcinomas and adenomas (Kluwe et al., 1985; Astill et al., 1996) and were suggested to be toxic to kidneys, thyroid and to the neuroendocrine system (Erkekoglu et al., 2012a,b; Liu et al., 2014). BPA was shown to alter mammary gland development and increase the incidence of tumors in Sprague-Dawley rats (Soto et al., 2013), causes development of breast, prostate, and nipple cancers (Keri et al., 2007), alter the development of reproductive organs, the testosterone excretion, and the sperm production (Richter et al., 2007). Heavy metals have also been recognized as likely inducers of testicular damage, although the mechanisms of testicular toxicity exerted by heavy metals are still unclear (De Toni et al., 2017). Several epidemiological studies showed associations between heavy metal exposure and adverse pregnancy outcomes (Motawei et al., 2013; Jameil, 2014; Quansah et al., 2015).

In this perspective, our work aims to evaluate the impact of microplastic, plastic additives and heavy metals contamination in the commercial fish species Lepidopus caudatus (Euphrasen, 1788) (Pisces, Trichiuridae), commonly known as silver scabbardfish (spatola in Italian), occurring in temperate waters of all oceans and in the Mediterranean Sea (D'Onghia et al., 2000). We have used the triple approach recently proposed by Fossi et al. (2018), a method that combines a measurement of marine waste and microplastic presence in organisms, the assessment of levels of plastic additives and persistent compounds, and their toxicological effects. This combined analysis allows a more robust assessment of the real impact of contaminants.

The study has been based on the following data: (1) analysis of the gastrointestinal content to evaluate the ingested microplastics; (2) quantitative and qualitative analysis of plastic additives and persistent compounds used as plastics tracers in the tissues of bioindicator organisms; (3) analysis of the toxicological effects of plastic additives ingestion through biomarkers detection and histological analysis. 


\section{MATERIALS AND METHODS}

For this study, 20 specimens (10 males and 10 females) of L. caudatus fished in FAO area 37 were analyzed. Fish were captured as part of local commercial fishing with longlines and transported to the laboratory where, in addition to taking samples for chemical and histological analyses, a check of the gastrointestinal contents was performed.

\section{Determination of Phthalates and Bisphenol A}

The extraction, performed by liquid-liquid method, was divided into different phases: aliquots of each sample (specifically muscle, gonad, gill, intestine and liver) were transferred into a glass tube with $2 \mathrm{ml} \mathrm{pH} 7$ of $0.1 \mathrm{M}$ phosphate buffer. After the addition of $3 \mathrm{ml}$ of dichloromethane, the samples have been shaken by vortex and centrifuged for $4 \mathrm{~min}$ at $3000 \mathrm{rpm}$. The organic phase was separated. The extraction was repeated with an additional $2 \mathrm{ml}$ of dichloromethane. The second extract was mixed with the previous one. After evaporation by nitrogen insufflation, the dried extracts were recovered with $50 \mu \mathrm{l}$ of methanol.

Analysis was performed by Ultra-High-Pressure Liquid Chromatography with Electro-Spray ionization and by Mass Quadrupole Mass Spectrometry. For this purpose, a Waters UHPLC-ESI-TQD Acquity system was used with the Acquity UPLC $^{\circledR}$ HSS C18 $1.8 \mu \mathrm{m}-2.1 \times 150 \mathrm{~mm}$ column and mobile phase consisting in water and methanol (both added at $0.1 \%$ with formic acid) for ESI+ analysis and water and methanol (both added at $0.1 \%$ with ammonia) for ESI- analysis, in percentages of gradient variables during the race. The mass spectrometry settings were as follows: capillary energy was $3.0 \mathrm{kV}$, source temperature was $150^{\circ} \mathrm{C}$, column temperature was $40^{\circ} \mathrm{C}$, desolvation temperature was $500^{\circ} \mathrm{C}$, desolvation gas was $1000 \mathrm{~L} / \mathrm{hr}$ and cone gas was $100 \mathrm{~L} / \mathrm{hr}$. The injected sample was 5 microliters.

The reading was performed using the MRM acquisition method, selecting the ionic transitions from the values of $\mathrm{m} / \mathrm{z}$ (ratio between the mass of the single ion fragment and its electric charge) obtained from the analysis of suitable reference materials and shown in Table 1.

Principal Components Analysis (PCA) on the distance matrix was used to identify in which tissue the phthalates presence was highest. Data were standardized before analysis and the results were displayed in a biplot distance (Legendre and Legendre, 2012).

The PCA on the correlation matrix was instead used to evaluate if exist a correlation between the various levels of phthalates. Data were standardized before analysis and the results were visualized in a biplot correlation (Turchetti et al., 2013).

\section{Trace Elements Analysis}

Arsenic (As), cadmium (Cd), cobalt (Co), chromium (Cr), copper $(\mathrm{Cu})$, lead $(\mathrm{Pb})$, mercury $(\mathrm{Hg})$, manganese $(\mathrm{Mn})$, nickel $(\mathrm{Ni})$, vanadium $(\mathrm{V})$, selenium $(\mathrm{Se})$, antimony $(\mathrm{Sb})$ and zinc $(\mathrm{Zn})$, were extracted and quantified in muscle of fish according to the method described in Copat et al. (2018). Briefly, aliquots of $0.5 \mathrm{~g}$ of muscle tissue were removed and acid digested in a
TABLE 1 | MRM acquisition method.

\begin{tabular}{lccc}
\hline Parametri UPLC-MS/MS & ESI & Sample 1 & Sample 2 \\
\hline DIDP & + & $447>141$ & $447>288.8$ \\
DINP & + & $419.2>127$ & $419.2>274$ \\
DEHP & + & $390.7>148.7$ & $390.7>112.9$ \\
Bis-benzilestere ftalato & + & $346.6>180.8$ & $346.6>238$ \\
Bis-butilestereftalato & + & $279.2>148.8$ & $279.2>204.7$ \\
Mono-N-octilestere ftalato & + & $278.9>148.8$ & $278.9>71$ \\
Mono-N-butilestere ftalato & + & $222.7>148.8$ & $222.7>204.8$ \\
Mono-metilestere ftalato & + & $180.7>148.7$ & $180.7>162.7$ \\
BPA & - & $226.8>132.8$ & $226.8>211.7$ \\
\hline
\end{tabular}

microwave system (Ethos Touch Control, Milestone S.r.l., Italy) equipped with pressurized vessels, using a digestion solution of $6 \mathrm{ml}$ of $65 \%$ nitric acid $\left(\mathrm{HNO}_{3}\right.$ ) (Carlo Erba) and $2 \mathrm{ml}$ of $30 \%$ peroxide hydrogen $\left(\mathrm{H}_{2} \mathrm{O}_{2}\right.$-Carlo Erba). The quantification of metals was carried on with an ICP-MS Elan-DRC-e (PerkinElmer, United States). Analytical blanks, standard and samples were processed with the same acid matrix. Standards for the instrument calibration were prepared with a multi-elements certified reference solution ICP Standard (Merck).

The method detection limits (MDL) estimated with ten blanks was calculated according to the following equation:

$$
\mathrm{MDL}=\text { One } \text {-tailed student } t \text {-test }(p=0.99 \% ; \mathrm{df}=\mathrm{n}-1) \times \mathrm{Sr}
$$

MDL ( $\mathrm{mg} / \mathrm{kg} \mathrm{ww}$ ) estimated for each trace elements are the following: As 0.013, Cd 0.002, Co 0.008, Cr 0.003, Cu $0.005, \mathrm{~Pb} 0.001$, Hg 0.0025, Mn 0.005, Ni 0.007, V 0.025, Se 0.03 , and $\mathrm{Zn} 0.109$.

A laboratory-fortified matrix (LFM) was processed for the quality control with recovery rates between 91.5 and $110 \%$.

Statistical analysis was performed with the software SPSS (version 20.0, Inc., IBM, United States). The normal distribution was verified using the Kolmogorov-Smirnov test. Since several trace element concentrations did not have a normal distribution, the Mann-Whitney non-parametric test was used to compare median concentrations between tissues.

\section{Histological Analysis}

Samples were fixed in $4 \%$ formaldehyde (Bio-Optica) in PBS buffered to $0.1 \mathrm{M}, \mathrm{pH} 7.4$ (Sigma Life Science) at room temperature for $36 \mathrm{~h}$. Gills were decalcified, prior to processing, with a decalcifier agent (Biodec R, Bio-Optica) for $3 \mathrm{~h}$ at room temperature. Histological examinations were performed according to our standard laboratory procedures (Brundo et al., 2011; Droutsa et al., 2019), and processed with Tissue Processing Center TPC 15 Duo (MEDITE $\left.{ }^{\circledR}\right)$. The sections were stained with Haematoxylin-Eosin (HE) (Bio-Optica) and observed under optical microscope (Leica DM750, Monument, CO, United States) equipped with a digital camera (Leica DFC500, Monument, CO, United States).

\section{Immunohistochemical Analysis}

The immunohistochemical protocol was performed on the liver and gonads sections to detect mouse monoclonal anti-VGT 
(Abcam, 1:1000) and on liver sections to detect mouse polyclonal anti-MT1 (Abcam, 1:1000); secondary antibody used is FITconjugated goat anti-mouse IgG (Sigma-Aldrich, 1:1000). Analysis were performed according to our standard laboratory procedures (Salvaggio et al., 2017; Pecoraro et al., 2018). Slides after mounted with mounting medium containing DAPI (Vectashield, Vector Laboratories), were observed with fluorescent microscope (Olympus Optical U-Ulh, Gilroy, CA, United States).

\section{RESULTS AND DISCUSSION}

The stomach content of the collected specimens contained highly digested food items, and in some specimens, we have found the bait fish (Sardina pilchardus). We failed to detect the presence of microplastics in the gastrointestinal tract of the fish analyzed, but chemical analysis revealed the presence of a high content of phthalates, and in particular high quantities of DIDP, DEHP, bis-benzylester phthalate, bis-butyl ester phthalate and mono-Nbutyl ester phthalate (Table 2).

The distance biplot (PC1 and PC2 were 53 and 25\%, respectively) showed that intestine is the most affected by the presence of DEHP, DIDP, bis-benzilesterephthalate, bisbutilesterephthalate, mono-N-butilesterephthalate and DINP (Figure 1). Instead, BPA and mono-metilesterephthalates were strongly present in gill. Otherwise, muscle, gonad and liver showed low levels of phthalates and BPA (Figure 1).

Correlation biplot (PC1 and PC2 were 53 and 25\%, respectively) showed simultaneous presence in samples of DEHP,

TABLE 2 | Results of chemical analysis performed with UPLC-ESI-TQD.

\begin{tabular}{lccccc}
\hline Analyte & Muscle & Liver & Gonad & Gill & Intestine \\
\hline DIDP & 9.0 & 14.5 & 10.8 & $<17.9 \underline{\mathrm{a}}$ & 41.1 \\
DINP & $3.7 \underline{\mathrm{a}}$ & $<10.2 \underline{\mathrm{a}}$ & $<6.8 \underline{\mathrm{a}}$ & $<17.9 \underline{\mathrm{a}}$ & $<18.5 \underline{\mathrm{a}}$ \\
DEHP & 43.3 & 20.6 & 30.5 & 93.6 & 193.0 \\
Bis-benzilestere ftalato & $<3.7 \underline{\mathrm{a}}$ & $<10.2 \underline{\mathrm{a}}$ & 10.5 & $<17.9 \underline{\mathrm{a}}$ & 33.3 \\
Bis-butilestereftalato & 13.0 & $<10.2 \underline{\mathrm{a}}$ & 8.5 & 29.6 & 77.8 \\
Mono-N-octilestere ftalato & 12.4 & $<10.2 \underline{\mathrm{a}}$ & 7.3 & $<17.9 \underline{\mathrm{a}}$ & $<18.5 \underline{\mathrm{a}}$ \\
Mono-N-butilestere ftalato & 9.9 & $<10.2 \underline{\mathrm{a}}$ & $<6.8 \underline{\mathrm{a}}$ & $<17.9 \underline{\mathrm{a}}$ & 54.8 \\
Mono-metilestere ftalato & $<3.7 \underline{\mathrm{a}}$ & $<10.2 \underline{\mathrm{a}}$ & $<6.8 \underline{\mathrm{a}}$ & 88.2 & $<18.5 \underline{\mathrm{a}}$ \\
BPA & $<3.7 \underline{\mathrm{a}}$ & 24.5 & 19.6 & 65.7 & $<18.5 \underline{\mathrm{a}}$ \\
\hline
\end{tabular}

a Limit of detection.

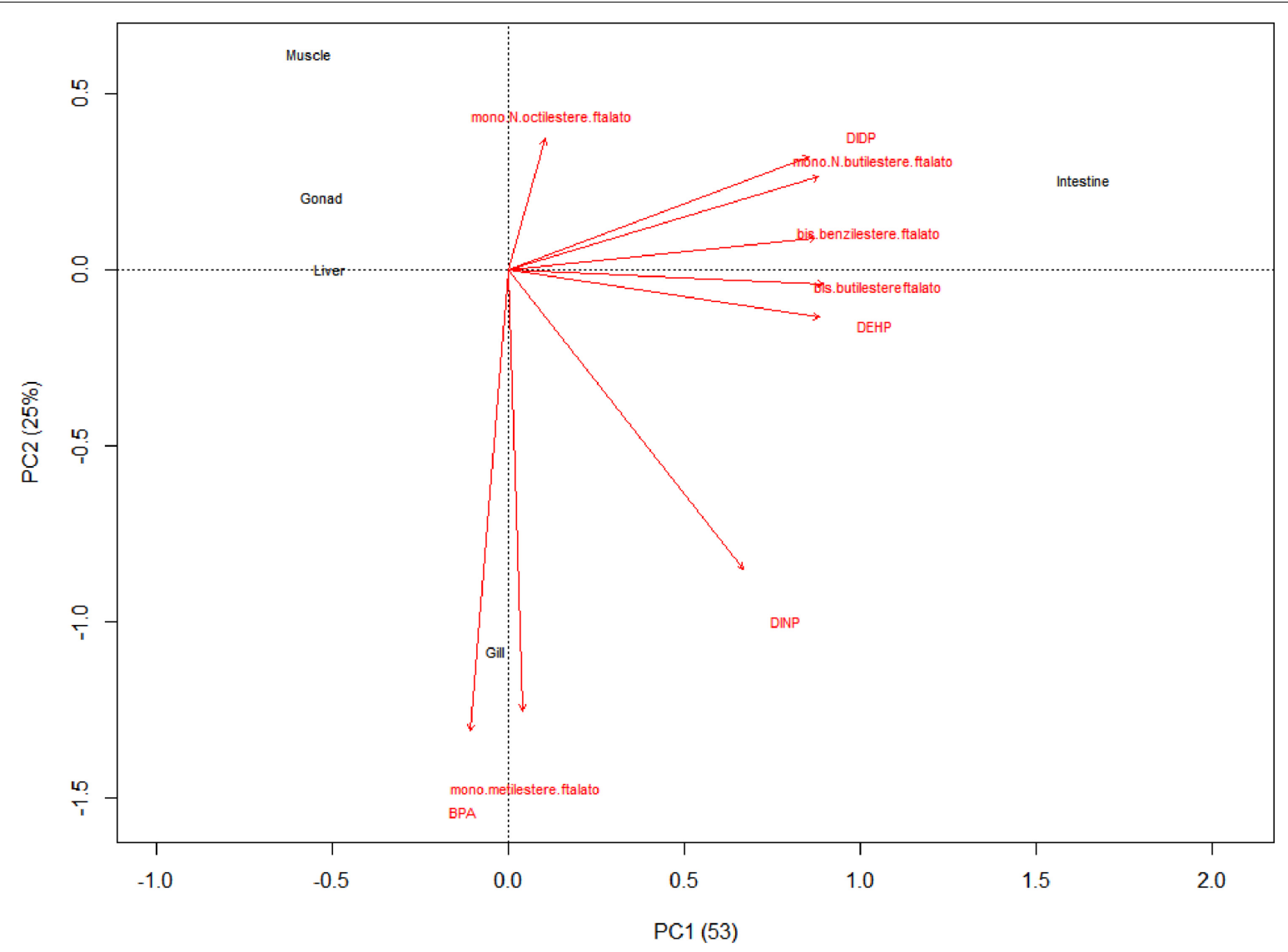

FIGURE 1 | The distance biplot showed that intestine is the most affected to presence of DEHP, DIDP, bis-benzilesterephthalate, bis-butilesterephthalate, mono-N-butilesterephthalate and DINP. Instead, BPA and mono-metilesterephthalates were strongly present in gill. Otherwise, muscle, gonad and liver showed low levels of phthalates and BPA. 


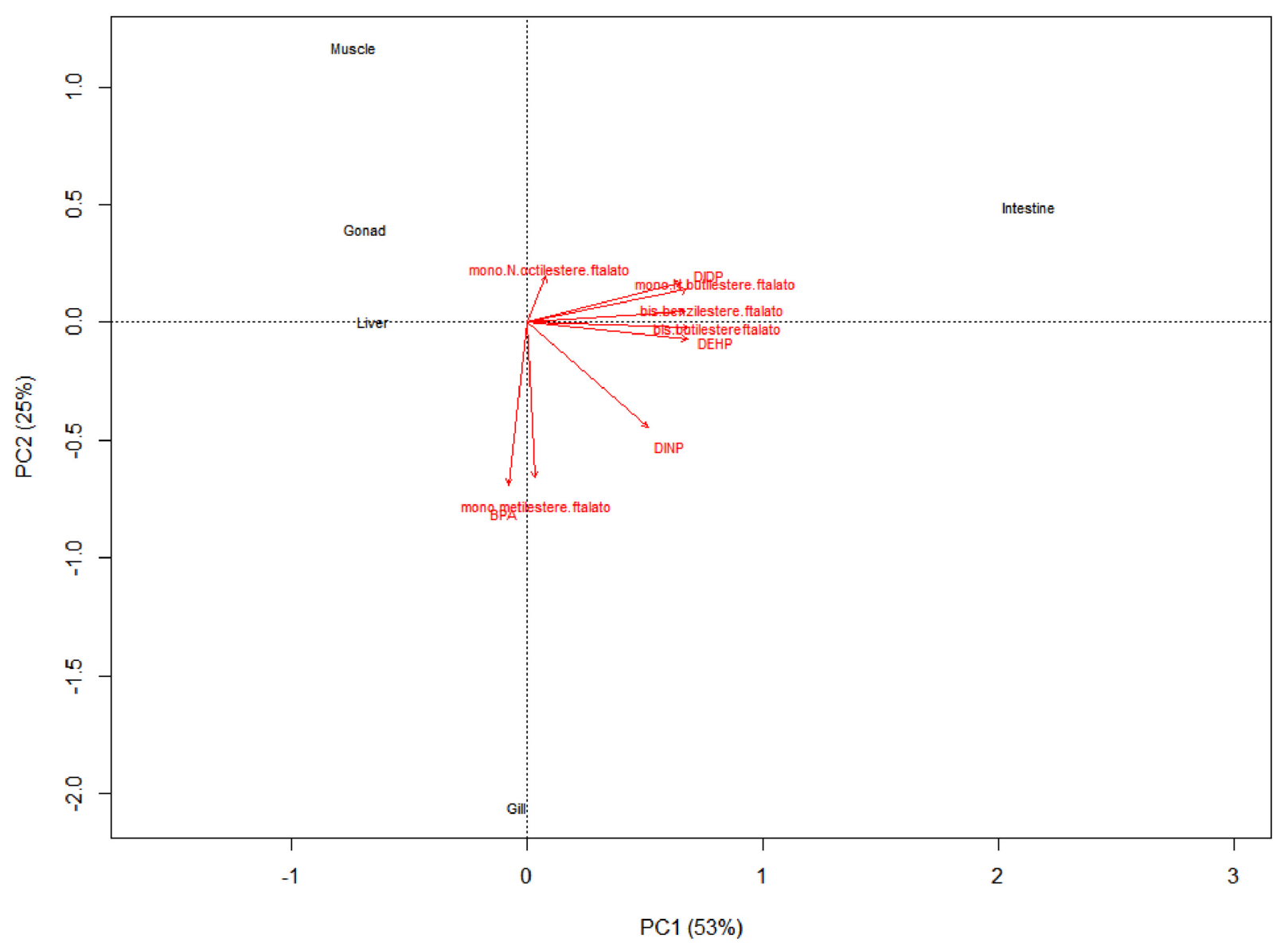

FIGURE 2 | Correlation biplot showed simultaneous presence of DEHP, DIDP, bis-benzilesterephthalate, bis-butilesterephthalate and mono-N-butilesterephthalate while BPA was related to presence of mono-metilesterephthalate. Otherwise, DINP and mono-N-octilesterephthalate were weakly related to others.

DIDP, bis-benzilesterephthalate, bis-butilesterephthalate and mono-N-butilesterephthalate, while BPA was related only to the presence of mono-metilesterephthalate (Figure 2). Otherwise, DINP and mono-N-octilesterephthalate were weakly related to others (Figure 2).

Our results are mostly in line with the data present in the literature for other aquatic species, except for DEHP, whose concentration is higher in our samples, especially in the liver. Indeed, DEHP and MEHP concentration were reported in ranges of 9-14.62 ng/g and 1.5-6.30 ng/g, respectively. Instead, according to our data, BPA concentrations in fishes varied from 2 to $75 \mathrm{ng} / \mathrm{g}$ in the liver and 1-11 ng/g in the muscle (Belfroid et al., 2002; Fossi et al., 2012; Valton et al., 2014; Guerranti et al., 2016). Probably, the low concentration or absence of contaminants in muscle, gonad and liver could be explained by the strong conversion of these substances to their metabolites after their assimilation (Sackett et al., 2013; Chattopadhyay and Chattopadhyay, 2015). The liver first passage after ingestion convert largely the substances in their metabolites before they reach the other districts. The high concentrations of BPA in gills seems to be related to a major exposure of this substance to the gills' surface.
These results could be explained by a recent exposure to BPA and, mainly, to phthalates. Since these free substances are largely degraded by marine environment, the high levels of them in fish could be related or to ingestion of microplastics, widely present in marine waters after accumulation and resuspension of plastic debris on the seabed, and the consequent slow releasing of them compounds (Paluselli et al., 2018) or, since these compounds are present in relatively high concentrations in marine water, they can be absorbed through the gills (Barboza et al., 2018).

Trace elements detected in tissue revealed a trend of concentrations generally higher in liver and intestine than in gill and muscle tissues, with the exception of few elements (Table 3 and Figure 3).

In particular, As and Cd were found significantly higher in liver and intestine than gill and muscle tissue $(p<0.001$ and $p<0.05$, respectively), although the liver concentrations had a wider value distribution. $\mathrm{Co}, \mathrm{Cu}, \mathrm{Pb}, \mathrm{V}$, and $\mathrm{Zn}$ were found with comparable median concentrations in gill, liver and intestine, significantly higher than muscle $(p<0.05)$. Median concentrations of $\mathrm{Cr}, \mathrm{Hg}$ and Se were comparable in all tissues analyzed, although $\mathrm{Hg}$ and $\mathrm{Se}$ had a wide value 
TABLE 3 | Descriptive statistic of trace elements ( $\mathrm{mg} / \mathrm{kg}$ wet weight) in the analyzed tissues.

\begin{tabular}{|c|c|c|c|c|c|c|c|c|c|c|c|c|}
\hline Gill & As & Cd & Co & $\mathrm{Cr}$ & $\mathrm{Cu}$ & $\mathrm{Pb}$ & $\mathrm{Hg}$ & Mn & $\mathrm{Ni}$ & $\mathrm{Zn}$ & $\mathrm{Se}$ & v \\
\hline Min & 0.078 & $<0.002$ & 0.082 & 0.752 & 0.585 & 0.191 & $<0.025$ & 5.058 & 0.115 & 10.21 & 0.195 & 0.421 \\
\hline Max & 1.666 & 0.055 & 1.114 & 2.460 & 5.375 & 6.403 & 0.043 & 169.3 & 2.867 & 28.70 & 0.592 & 7.219 \\
\hline Median & 0.384 & 0.006 & 0.140 & 0.896 & 3.490 & 0.833 & 0.027 & 21.92 & 1.834 & 20.13 & 0.321 & 1.312 \\
\hline Mean & 0.510 & 0.015 & 0.260 & 1.154 & 2.799 & 1.938 & 0.029 & 38.28 & 1.708 & 19.16 & 0.355 & 2.019 \\
\hline SD & 0.533 & 0.018 & 0.349 & 0.578 & 1.787 & 2.352 & 0.006 & 53.74 & 0.973 & 5.693 & 0.132 & 2.208 \\
\hline \multicolumn{13}{|l|}{ Liver } \\
\hline Min & 0.038 & 0.004 & 0.065 & 0.492 & 1.236 & 0.056 & $<0.025$ & 1.786 & 0.011 & 6.660 & 0.230 & 0.131 \\
\hline Max & 2.295 & 0.195 & 0.312 & 0.940 & 4.032 & 1.456 & 0.218 & 10.04 & 0.462 & 34.14 & 12.84 & 14.01 \\
\hline Median & 1.122 & 0.039 & 0.074 & 0.629 & 3.717 & 0.353 & 0.045 & 3.602 & 0.149 & 16.53 & 0.690 & 0.633 \\
\hline Mean & 1.056 & 0.080 & 0.149 & 0.686 & 2.488 & 0.621 & 0.092 & 4.377 & 0.200 & 17.02 & 4.271 & 4.696 \\
\hline $\mathrm{SD}$ & 0.893 & 0.088 & 0.121 & 0.207 & 1.027 & 0.610 & 0.089 & 3.142 & 0.187 & 9.585 & 5.875 & 6.631 \\
\hline \multicolumn{13}{|l|}{ Intestine } \\
\hline Min & 0.894 & 0.013 & 0.194 & 0.736 & 2.400 & 0.873 & 0.031 & 27.25 & 0.355 & 11.83 & 0.355 & 0.943 \\
\hline Max & 1.473 & 0.030 & 0.415 & 2.098 & 3.346 & 3.652 & 0.044 & 54.39 & 0.976 & 26.18 & 0.556 & 3.587 \\
\hline Median & 1.174 & 0.020 & 0.369 & 1.052 & 2.813 & 1.907 & 0.040 & 52.07 & 0.701 & 13.81 & 0.468 & 2.811 \\
\hline Mean & 1.180 & 0.021 & 0.326 & 1.295 & 2.853 & 2.144 & 0.038 & 44.57 & 0.677 & 17.27 & 0.459 & 2.447 \\
\hline SD & 0.259 & 0.008 & 0.104 & 0.637 & 0.424 & 1.256 & 0.006 & 13.46 & 0.278 & 6.954 & 0.090 & 1.216 \\
\hline \multicolumn{13}{|l|}{ Muscle } \\
\hline Min & 0.039 & $<0.002$ & 0.004 & 0.535 & 0.137 & 0.005 & $<0.025$ & 0.150 & $<0.007$ & 1.056 & 0.054 & $<0.025$ \\
\hline $\operatorname{Max}$ & 0.186 & 0.011 & 0.021 & 1.035 & 0.360 & 0.043 & 0.090 & 2.445 & 0.066 & 7.913 & 0.189 & 0.419 \\
\hline Median & 0.118 & 0.002 & 0.011 & 0.780 & 0.257 & 0.015 & 0.025 & 0.487 & 0.013 & 2.594 & 0.153 & $<0.025$ \\
\hline Mean & 0.114 & 0.003 & 0.011 & 0.728 & 0.257 & 0.018 & 0.042 & 0.722 & 0.027 & 3.406 & 0.147 & 0.080 \\
\hline SD & 0.055 & 0.003 & 0.005 & 0.175 & 0.065 & 0.012 & 0.027 & 0.768 & 0.023 & 2.467 & 0.039 & 0.131 \\
\hline
\end{tabular}

distribution in liver as well as $\mathrm{Cr}$ in the intestine. Mn had the highest median concentrations in intestine $(p<0.01$ vs. other tissues), followed by gill ( $p<0.01$ vs. liver and muscle) and liver ( $p<0.05$ vs. muscle). Ni had the highest median concentrations in gill ( $p<0.01$ vs. other tissues) followed by intestine ( $p<0.05$ vs. liver and muscle).

Several metals represent chemicals known to be used in the plastic industry as additive. Recently, $\mathrm{Pb}$ has been phased out as a plastic additive in Europe (Murphy, 2001), while Cd is still under discussion, and is used as pigment, heat and UV-stabilized in PVC. It has been suggested that metals are mostly absorbed onto the pellet surface and are therefore likely bioavailable by ingestion (Ashton et al., 2010). Today the use of some of these metals is restricted, for example As, that is used as antimicrobial and plasticizer (Cordeiro et al., 2012). Thus, contamination of food sources, whether by naturally occurring or introduced toxins, is a concern for consumers due to the adverse health effects that have been associated with exposure to such compounds (Piazzolla et al., 2015; Scanu et al., 2016; Pappalardo et al., 2017; Ferrante et al., 2018a,b).

Few studies in literature report trace element concentrations in L. caudatus from the Mediterranean Sea. Naccari et al. (2015) evaluated the presence of $\mathrm{Pb}, \mathrm{Cd}$ and $\mathrm{Hg}$ in specimens collected from the Tyrrhenian and Ionian Seas. They observe the absence of residual levels of toxic metals analyzed in muscle, with concentrations always below the limit of detection, with the exception of $\mathrm{Cd}$ and $\mathrm{Hg}$ in samples from the Ionian Sea, where they revealed mean concentrations of 0.045 and $0.458 \mathrm{mg} / \mathrm{kg}$ ww respectively. Comparable $\mathrm{Hg}$ concentrations have been found by Storelli et al. (2007). The authors reported a mean value of $0.59 \mathrm{mg} / \mathrm{kg}$ ww in the muscle tissue of L. caudatus caught during the summer. These results are higher than concentrations reported in this study (Cd $0.003 \mathrm{mg} / \mathrm{kg}$, $\mathrm{Hg} 0.042 \mathrm{mg} / \mathrm{kg}$ ), but they could be influenced by sampling season and by the biological stage of the sampled specimens (Copat et al., 2012). Conversely, Lo Turco et al. (2013) reported metal concentrations in muscle and gills of L. caudatus, collected during the winter in the Strait of Messina, which are significantly lower (for all the analyzed metals but not for Cd and As) than concentrations detected in this study, especially in gills. Similarly, to our findings, they found higher metals concentrations in gills respect to muscle tissue, with the exception of As.

Histological analysis did not reveal any morphological alteration of the gills, liver and gonads in both sexes: liver and gonads maintain their typical organization (Figures 4-6); however, immunohistochemical analysis for anti-metallothionein 1 antibody showed a strong positivity of liver cells, both in females (Figures 7A-C) and males (Figures 7D-F) in all 20 the samples analyzed, showing a strong stress that activated a cell detoxification system.

Induction of MTs synthesis, as evidenced with different techniques, is considered a specific and highly sensitive response to heavy metal pollution (Sindermann, 1995; Viarengo et al., 1999, 2000; Cosson, 2000; Amaral et al., 2002). Amaral et al. (2002), for example, found in samples of Scophthalmus maximus exposed to different concentrations of cadmium, a significant increase of MTs syntesis in fish liver occurred that was dependent 

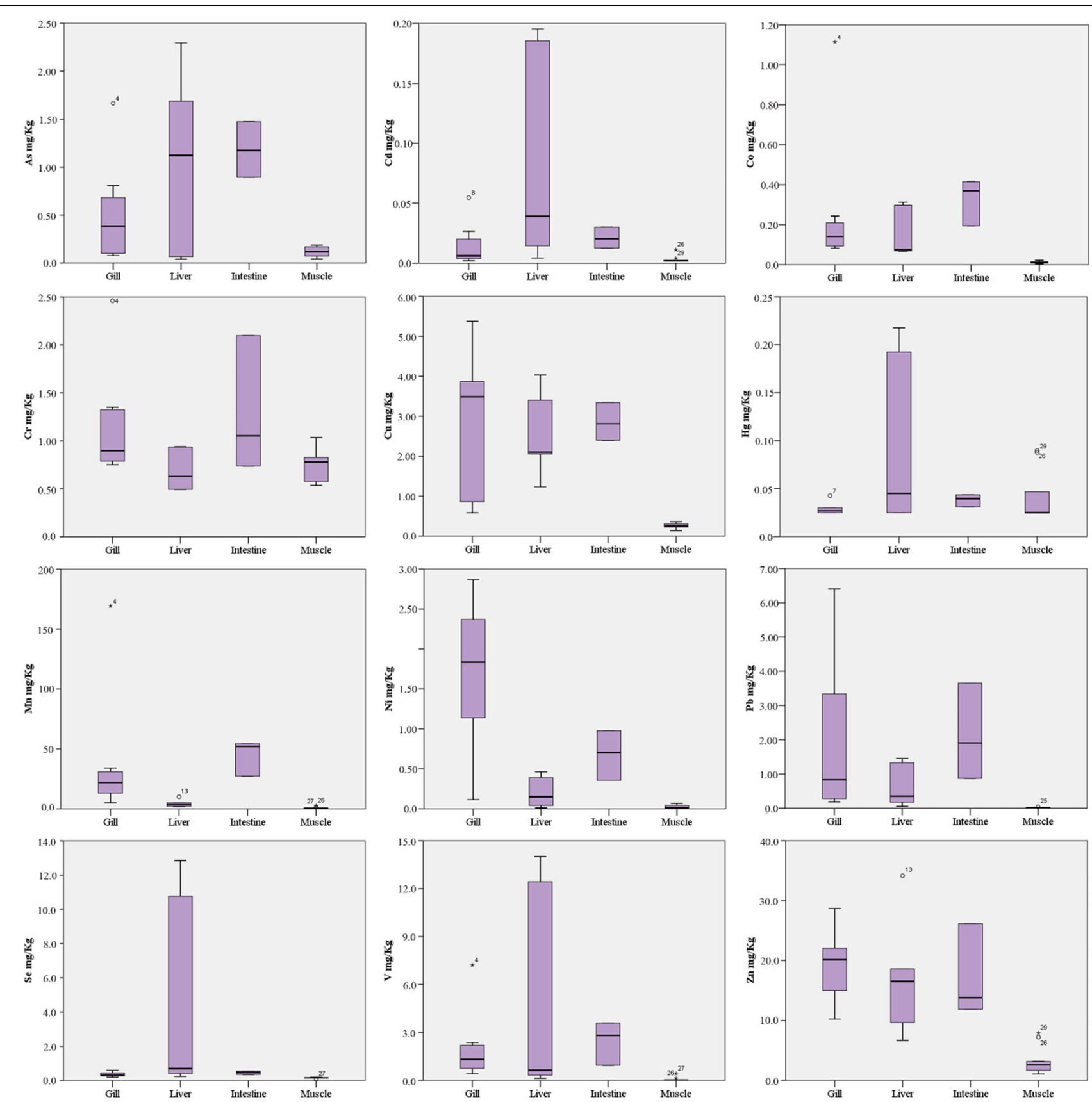

FIGURE 3 | Box plots of trace elements concentrations (mg/Kg ww) in fish tissues.

on the cadmium concentration and the exposure time. Authors in fact suggest that MT immunohistochemistry are good tools for clarifying metal and MTs tracking routes in hepatocytes which renders them useful biomarkers of metal exposure.

The immunohistochemical analysis for the anti-vitellogenin antibody showed in females a strong positivity both in the liver cells (Figures 8A-C), and in the gonads (Figure 9), as we expected. The analysis of the liver and gonadal preparations of the male specimens was found to be always negative except for a specimen, in which it was highlighted a positivity in some areas of the liver (Figures 8D-F) and in two areas at the level of the gonad in the intermediate portion (Figure 10). These results show that the specimen has been exposed to contaminants that can act as endocrine disruptors, activating the gene expression for vitellogenin, which in males and immature females is normally silenced (Matozzo et al., 2008).

The presence of plastic additives and heavy metals could be related to ingestion, accumulation and low degradation of microplastics as it has been documented in marine organisms belonging to different species and with different feeding habits. 


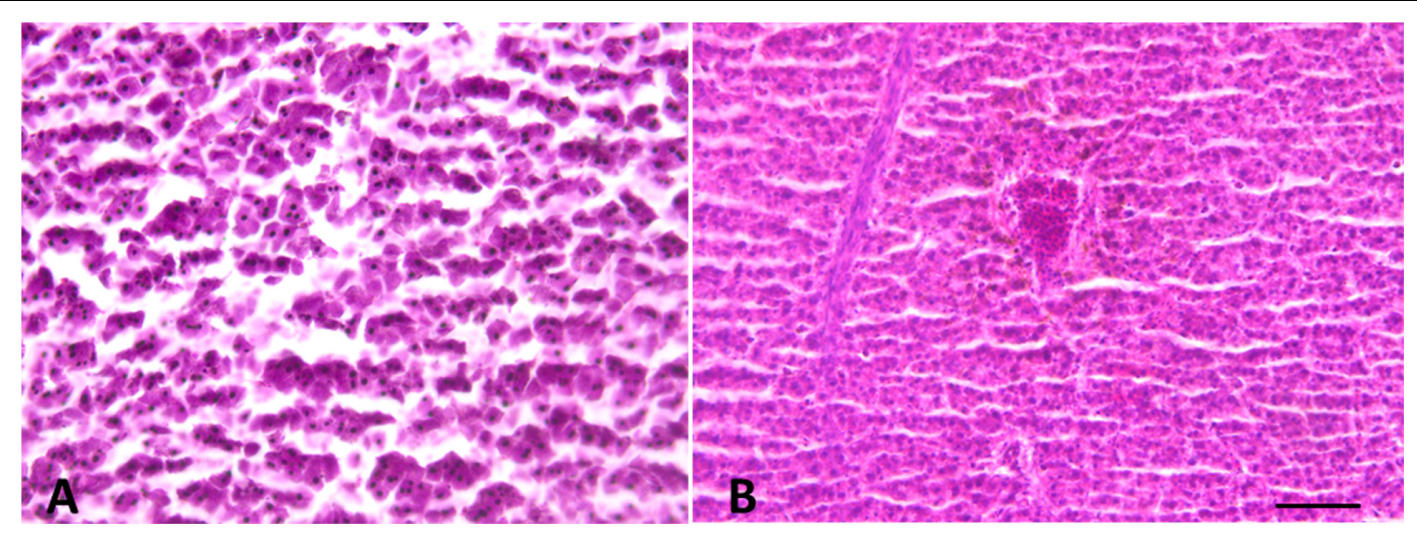

FIGURE 4 | Liver morphology images. (A) Male and (B) Female. Liver histology showing normal hepatocytes. Scale bar: 200 $\mu$ m.
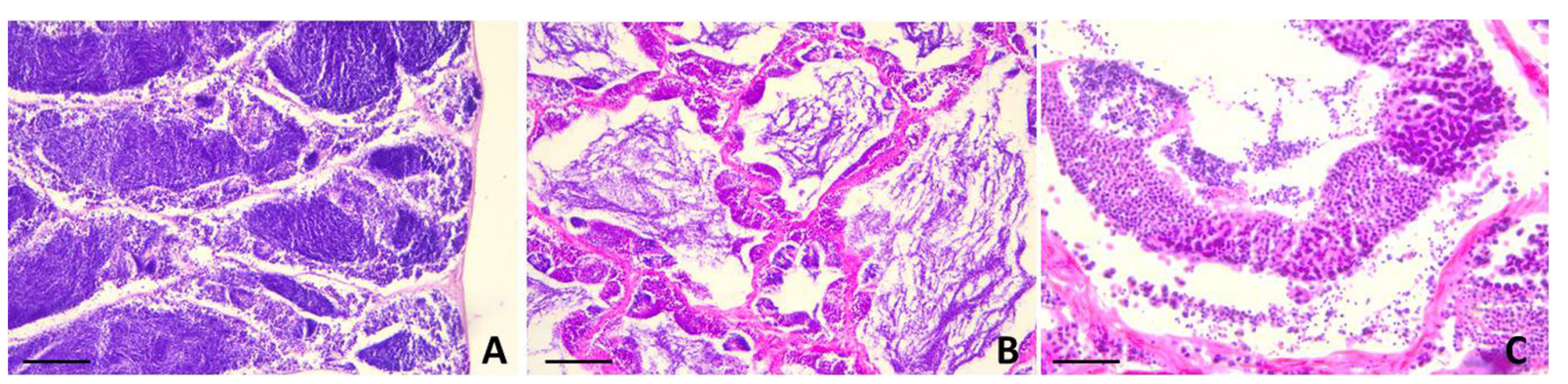

FIGURE 5 | Testis sections of L. caudatus. (A) anterior portion; (B) intermediate portion; (C) posterior portion. Sperm cells are visible at different stages of maturation. Scale bar: $200 \mu \mathrm{m}$.
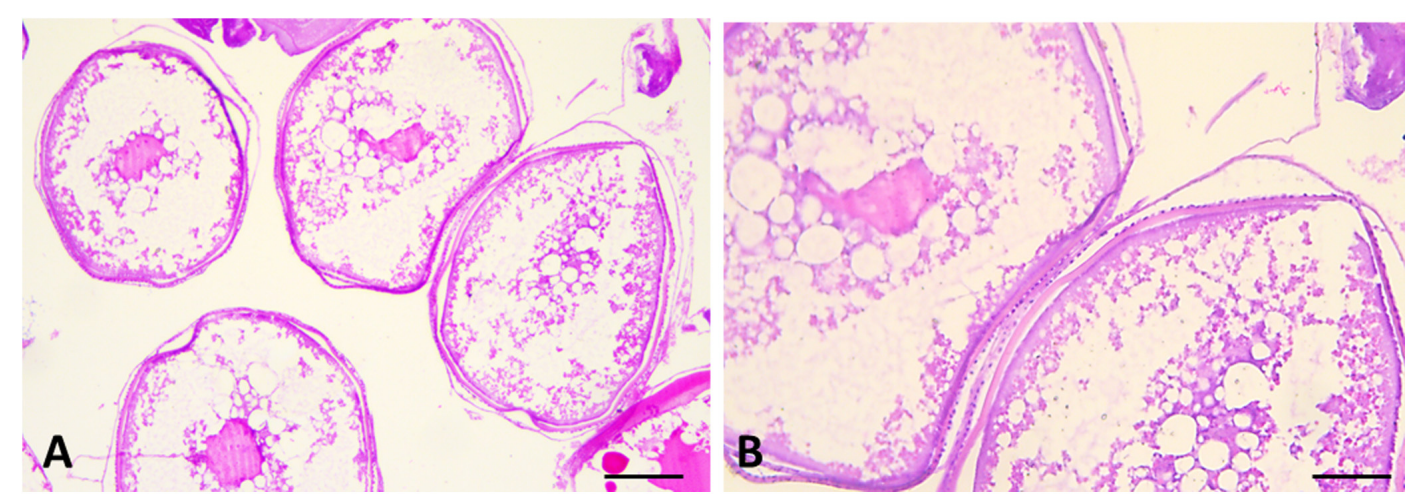

FIGURE 6 | Sections of female gonads of L. caudatus. Follicles in different stages of maturation are evident. Scale bar: A, $200 \mu \mathrm{m}$; B, $100 \mu \mathrm{m}$.

In fact, due to their small size, microplastics can be accidentally ingested during filtration or ingestion of prey. In particular, plastic particles have been found in more than 100 marine species, from zooplankton to whales, including marine reptiles and birds (Gallo et al., 2018). Marine organisms can ingest microplastics by mistaking them for prey (Cole et al., 2011; Campani et al., 2013; Wright et al., 2013; Romeo et al., 2016) or accidentally during feeding (Fossi et al., 2014). Once intaked, the microplastics accumulate in the intestine, move into other tissues or are expelled, depending on the size, shape and composition
(Gallo et al., 2018). In sea crab (Carcinus maenas) and in several filtering molluscs, it has been shown that not only they ingest the microplastics together with food, but these particles can remain entrapped in gills (Moore, 2008; Watts et al., 2014). Goldstein and Goodwin (2013) have assessed the potential effects of microplastic on the rafting community, examining the gastrointestinal tracts of 385 barnacles collected from the North Pacific Subtropical Gyre (NPSG) for evidence of plastic ingestion. They found that $33.5 \%$ of the barnacles had plastic particles present in their gastrointestinal tract. Their results suggest that 


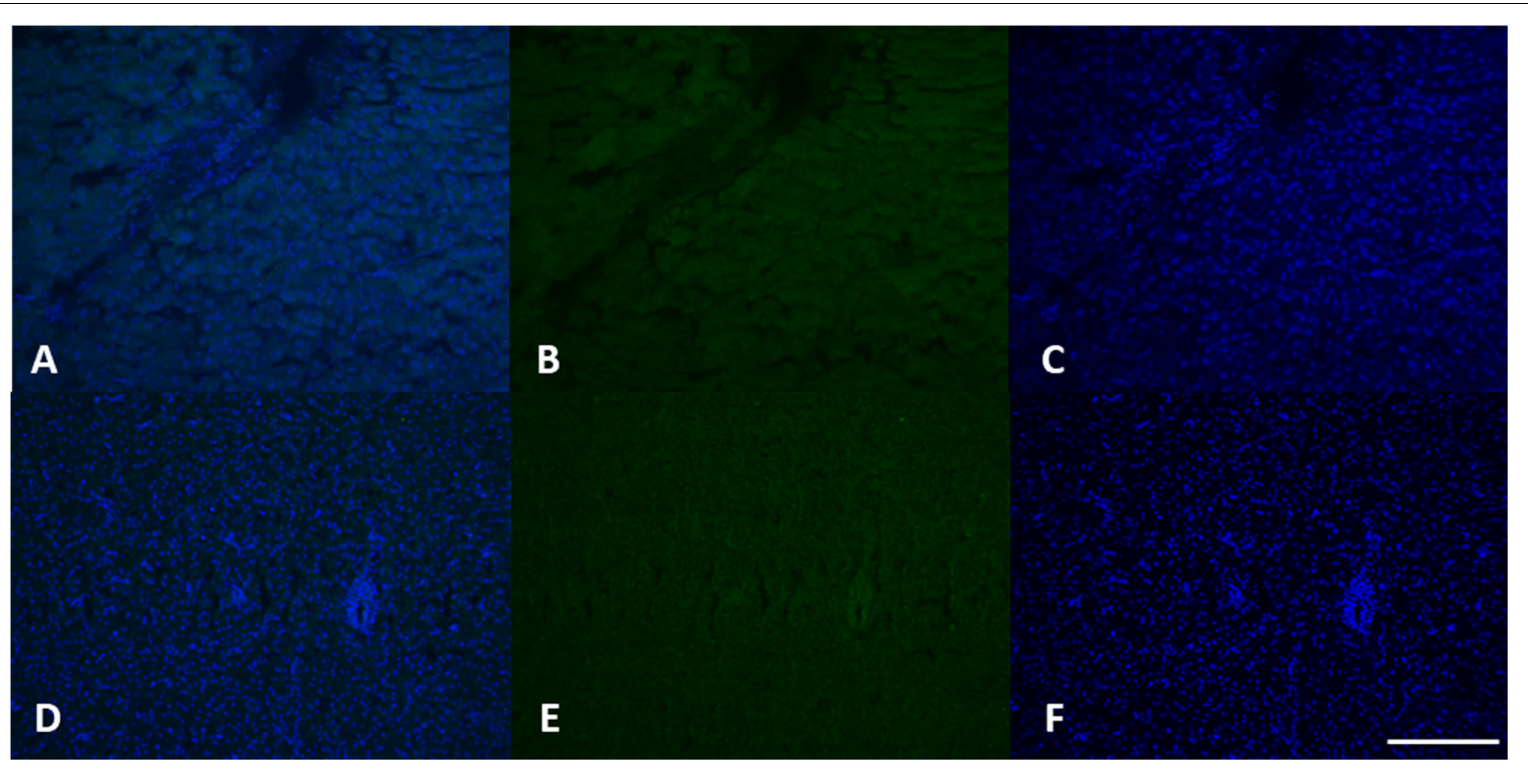

FIGURE 7 | MT 1 expression in female (A-C) and male (D-F) liver tissue. Using specific antibodies anti-MT1, it has been showed a clear expression of these proteins in the hepatocytes. DAPI staining for nuclei detection (blue). Scale bar: $200 \mu \mathrm{m} 2$.

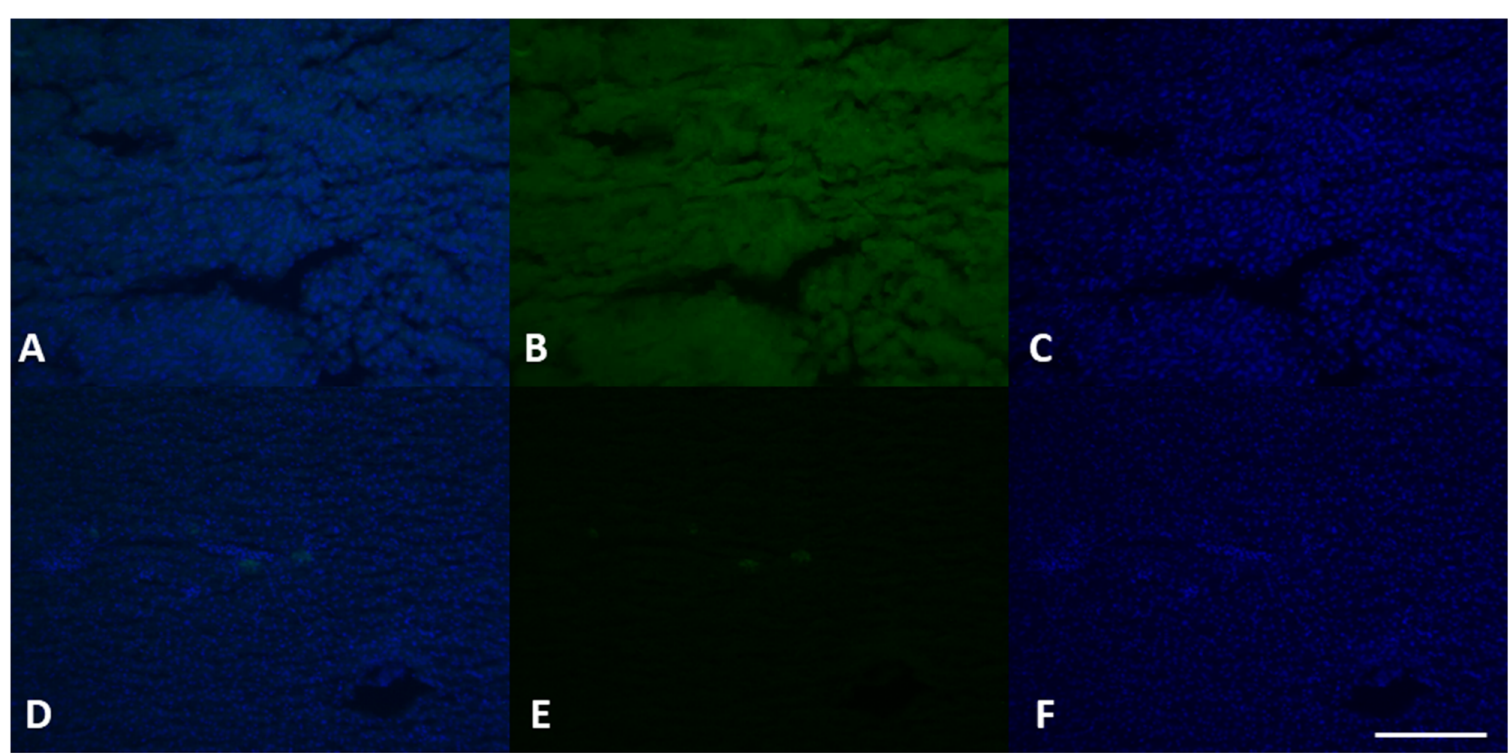

FIGURE 8 | VGT expression in female (A-C) and male (D-F) liver tissue. Using specific antibodies anti-VGT, it has been showed a clear expression of these proteins in the hepatocytes of female liver, but some positive areas are also evident in some areas of the liver in the male (green). DAPI staining for nuclei detection (blue).

Scale bar: $200 \mu \mathrm{m}$.

barnacle ingestion of microplastic is relatively common, but with unknown trophic impacts on the rafting community and the NPSG ecosystem.

Ingestion of microplastics has been verified also in the stomach of the Humboldt squid (Dosidicus gigas) (Braid et al., 2012), a large predator that usually feeds at depths between 200 and $700 \mathrm{~m}$, although the ingestion route is not yet clear.

Plastic fibers, probably derived from fibers of trawl nets and fragments of plastic bags, have been identified in the intestine of the Norway lobster, Nephrops norvegicus (Murray and Cowie, 2011): these organisms have various modes of feeding, including scavenging and predation, but they are not suitable for cutting flexible filamentary materials that are not eliminated by the normal digestive processes.

The results obtained confirm that the exposure to plastics additives of marine organisms is a widespread phenomenon and underlines the environmental relevance of the problem of plastic waste at sea. It is therefore urgent that scientific 


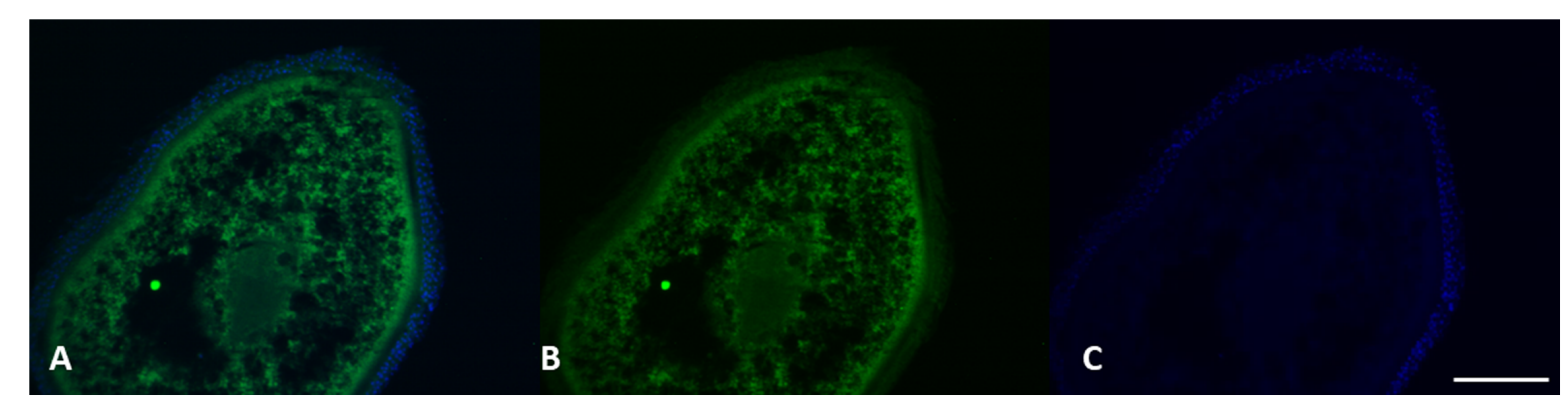

FIGURE 9 | VGT expression in female gonads of $L$. caudatus. It has been showed a clear expression of these proteins (green). DAPI staining for nuclei detection (blue). Scale bar: $200 \mu \mathrm{m}$

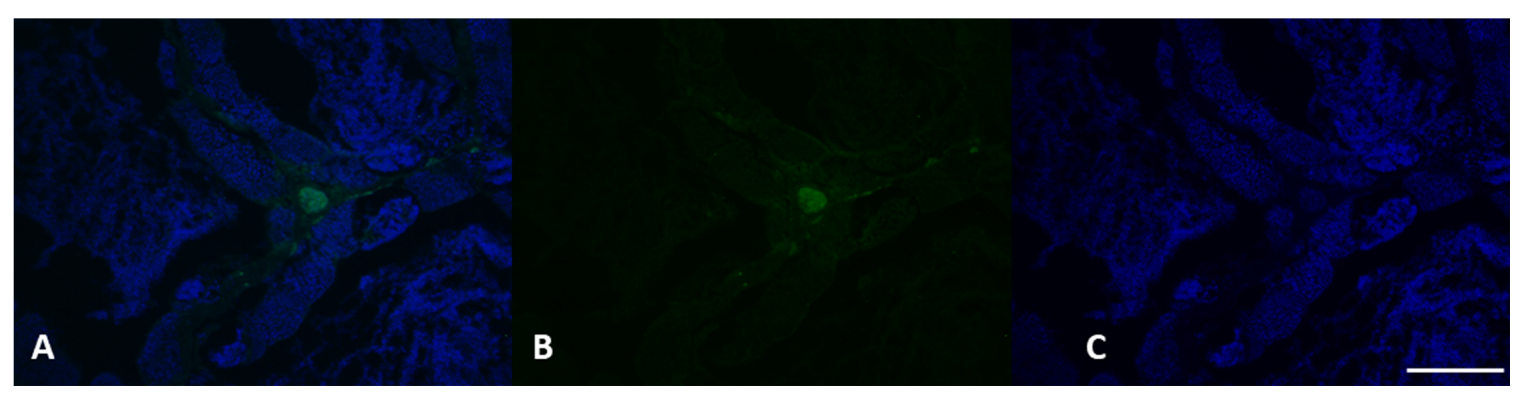

FIGURE 10 | VGT expression in testis of L. caudatus. It has been showed a clear expression of this protein in some areas (green). DAPI staining for nuclei detection (blue). Scale bar: $200 \mu \mathrm{m}$.

research acquires new knowledge and helps to raise awareness of this emerging issue.

Although the presence of microplastics in the intestine of the studied species could not be confirmed, the results obtained suggest an exposure of marine organisms to plastics additives (phthalates, bisphenol A and heavy metals), thus underlining the environmental relevance of the problem of plastic waste in the sea. It is therefore urgent that scientific research acquires new knowledge and helps to raise awareness of this emerging issue

\section{CONCLUSION}

The existence of microplastics and their potential impact on wildlife has received more public and scientific attention in recent years (Betts, 2008; Galloway, 2015; Lusher, 2015). Indeed, it is only with the recent European (Marine Strategy Directive Marine Strategy Directive [MSFD, 2008/56/EC], 2008) that the microplastics have been inserted among the contaminants worth to be monitored. However, at the moment, any analytical method is reliable to investigate microplastics, in particular those having diameters smaller than $10 \mu \mathrm{m}$. In fact, these fragments, due to their ability to be adsorbed by digestive and respiratory systems and, consequently, to be largely distributed in all part of body, are the most dangerous for health (Powell et al., 2010; Wright and Kelly, 2017).

This study confirmed the presence and the potential endocrine disruption effect of chemicals used as and/or associated with plastic additives in L. caudatus, thus suggesting the potential bioaccumulation of microplastics in marine organisms and the serious threat for aquatic systems and human health. The results obtained provide a further contribution to the knowledge on the impact of plastic wastes and especially of associated contaminants on marine life and on fish species of commercial interest. This study also confirms the validity of the triple monitoring approach proposed by Fossi et al. (2018), and the role of vitellogenin as a valid biomaker of exposure to endocrine disruptors.

\section{AUTHOR CONTRIBUTIONS}

AS, FT, and MB developed the research idea and experimental design. FT, DM, and GM collected the specimens. AM, RP, and ES made histological and immunohistochemical analysis. $\mathrm{MB}$, CC, and PZ co-wrote the manuscript. EK, IG, RC, BL, and MF reviewed the manuscript. All authors read and approved the final manuscript.

\section{FUNDING}

This study was funded by the Department of Biological, Geological and Environmental Science and by the Department of Medical Sciences, Surgical and Advanced Technologies "G.F. Ingrassia” - Hygiene and Public Health, University of Catania. 


\section{REFERENCES}

Alessi, E., Di Carlo, G., Campogianni, S., Tangerine, B., and Pietrobelli, E. (2018). Out of the Plastic Trap: Saving the Mediterranean from Plastic Pollution. Roma: WWF Mediterranean Marine Initiative.

Amaral, A. F., Alvarado, N., Marigomez, I., Cunha, R., Hylland, K., and Soto, M. (2002). Autometallography and metallothionein immunohistochemistry in hepatocytes of turbot (Scophthalmus maximus L.) after exposure to cadmium and depuration treatment. Biomarkers 7, 491-500. doi: 10.1080/ 1354750021000034843

Anderson, J. C., Park, B. J., and Palace, V. P. (2016). Microplastics in aquatic environments: implications for Canadian ecosystems. Environ. Pollut. 218, 269-280. doi: 10.1016/j.envpol.2016.06.074

Ashton, K., Holmes, L., and Turner, A. (2010). Association of metals with plastic production pellets in the marine environment. Mar. Pollut. Bull. 60, 2050-2055. doi: 10.1016/j.marpolbul.2010.07.014

Astill, B. D., Gingell, R., Guest, D., Hellwig, J., Hodgson, J. R., Kuettler, K., et al. (1996). Oncogenicity testing of 2-ethylhexanol in Fischer 344 rats and B6C3F1 mice. Fundam. Appl. Toxicol. 31, 29-41. doi: 10.1006/faat.1996.0073

Avio, C. G., Gorbi, S., Milan, M., Benedetti, M., Fattorini, D., D’Errico, G., et al. (2015). Pollutants bioavailability and toxicological risk from microplastics to marine mussels. Environ. Pollut. 198, 211-222. doi: 10.1016/j.envpol.2014.12. 021

Avio, C. G., Gorbi, S., and Regoli, F. (2017). Plastics and microplastics in the oceans: from emerging pollutants to emerged threat. Mar. Environ. Res. 128, 2-11. doi: 10.1016/j.marenvres.2016.05.012

Barboza, L. G. A., Russo Vieira, L., Branco, V., Carvalho, C., and Guilhermino, L. (2018). Microplastics increase mercury bioconcentration in gills and bioaccumulation in the liver, and cause oxidative stress and damage in Dicentrarchus labrax juveniles. Sci. Rep. 8:15655. doi: 10.1038/s41598-01834125- $\mathrm{z}$

Belfroid, A., van Velzen, M., van der Horst, B., and Vethaak, D. (2002). Occurrence of bisphenol A in surface water and uptake in fish: evaluation of field measurements. Chemosphere 49, 97-103. doi: 10.1016/s0045-6535(02)00157-1

Betts, K. (2008). Why small plastic particles may pose a big problem in the oceans. Environ. Sci. Technol. 42, 8995-8995. doi: 10.1021/es802970v

Braid, H. E., Deeds, J., De Grasse, S. L., Wilson, J. J., Osborne, J., and Hanner, R. H. (2012). Preying on commercial fisheries and accumulating paralytic shellfish toxins: a dietary analysis of invasive Dosidicus gigas (Cephalopoda Ommastrephidae) stranded in pacific canada. Mar. Biol. 159, 25-31. doi: 10. 1007/s00227-011-1786-4

Brundo, M. V., Longo, G., Sottile, L., Trovato, M., Vitale, D., and Viscuso, R. (2011). Tizo morphological and ultrastructural organization of the spermatheca of some Tettigoniidae (Insecta, Orthoptera). Ital. J. Zool. 78, 53-62. doi: 10. 1080/11250003.2010.498448

Campani, T., Baini, M., Giannetti, M., Cancelli, F., Mancusi, C., Serena, F., et al. (2013). Presence of plastic debris in loggerhead turtle stranded along the tuscany coasts of the pelagos sanctuary for mediterranean marine mammals (Italy). Mar. Pollut. Bull. 74, 225-230. doi: 10.1016/j.marpolbul.2013. 06.053

Chattopadhyay, S., and Chattopadhyay, D. (2015). Remediation of DDT and its metabolites in contaminated sediment. Curr. Pollut. Rep. 1, 248-264. doi: 10.1007/s40726-015-0023-Z

Cole, M., Lindeque, P., Halsband, C., and Galloway, T. S. (2011). Microplastics as contaminants in the marine environment: a review. Mar. Pollut. Bull. 62, 2588-2597. doi: 10.1016/j.marpolbul.2011.09.025

Copat, C., Brundo, M. V., Arena, G., Grasso, A., Oliveri Conti, G., Ledda, C., et al. (2012). Seasonal variation of bioaccumulation in Engraulis encrasicolus (Linneaus, 1758) and related biomarkers of exposure. Ecotoxicol. Environ. Saf. 86, 31-37. doi: 10.1016/j.ecoenv.2012.09.006

Copat, C., Grasso, A., Fiore, M., Cristaldi, A., Zuccarello, P., Signorelli, S. S., et al. (2018). Trace elements in seafood from the mediterranean sea: an exposure risk assessment. Food Chem. Toxicol. 115, 13-19. doi: 10.1016/j.fct.2018.03.001

Cordeiro, F., Baer, I., Robouch, P., Emteborg, H., Charoud-got, J., Kortsen, K. B., et al. (2012). IMEP-34: Heavy Metals in Toys according to EN 71-3:1994 - EU Science Hub - European Commission. EU Science Hub. doi: 10.2787/63387 Available at: https://ec.europa.eu/jrc/en/publication/eur-scientific-and- technical-research-reports/imep-34-heavy-metals- toys-according-en-71$-31994$

Cosson, R. P. (2000). Bivalve metallothionein as a biomarker of aquatic ecosystem pollution by trace metals: limits and perspectives. Cell Mol. Biol. 46, 295-309.

Cozar, A., Sanz-Martin, M., Martì, E., González-Gordillo, J. I., Ubeda, B., Gálvez, J. Á., et al. (2015). Plastic accumulation in the mediterranean sea. PLoS One 10:e0121762. doi: 10.1371/journal.pone.0121762

Davarpanah, E., and Guilhermino, L. (2015). Single and combined effects of microplastics and copper on the population growth of the marine microalgae Tetraselmis chuii. Estuar Coast. Shelf Sci. 167, 269-275. doi: 10.1016/j.ecss.2015. 07.023

De Toni, L., Tisato, F., Seraglia, R., Roverso, M., Gandin, V., Marzano, C., et al. (2017). Phthalates and heavy metals as endocrine disruptors in food: a study on pre-packed coffee products. Toxicol. Rep. 4, 234-239. doi: 10.1016/j.toxrep. 2017.05.004

D’Onghia, G., Mastrototaro, F., and Maiorano, P. (2000). Biology of silver scabbard fish, Lepidopus caudatus (Trichiuridae), from the Ionian Sea (Easter-Central Mediterranean). Cybium 24, 249-262.

Droutsa, A., Emmanouil, N., Raptopoulos, K., Salvaggio, A., Mazzei, V., Pecoraro, R., et al. (2019). Trace elements bioaccumulation in Porcellionides pruinosus (Brandt, 1833) and related biomarkers of exposure. Microsc. Res. Tech. 82, 651-657. doi: 10.1002/jemt.23211

Engler, R. E. (2012). The complex interaction between marine debris and toxic chemicals in the ocean. Environ. Sci. Technol. 46, 12302-12315. doi: 10.1021/ es3027105

Erkekoglu, P., Giray, B. K., Kizilgun, M., Hininger-Favier, I., Rachidi, W., Roussel, A. M., et al. (2012a). Thyroidal effects of di-(2-ethylhexyl) phthalate in rats of different selenium status. J. Environ. Pathol. Toxicol. Oncol. 31, 143-153. doi: 10.1615/jenvironpatholtoxicoloncol.v31.i2.60

Erkekoglu, P., Giray, B. K., Kızilgün, M., Rachidi, W., Hininger-Favier, I., Roussel, A. M., et al. (2012b). Di(2-ethylhexyl)phthalate-induced renal oxidative stress in rats and protective effect of selenium. Toxicol. Mech. Methods 22, 415-423. doi: 10.3109/15376516.2012.666652

Erkekoglu, P., Zeybek, N. D., Giray, B., Asan, E., Arnaud, J., and Hincal, F. (2011) Reproductive toxicity of di(2-ethylhexyl) phthalate in selenium-supplemented and selenium-deficient rats. Drug Chem. Toxicol. 34, 379-389. doi: 10.3109/ 01480545.2010.547499

Ferrante, M., Pappalardo, A. M., Ferrito, V., Pulvirenti, V., Fruciano, C., Grasso, A., et al. (2017). Bioaccumulation of metals and biomarkers of environmental stress in Parablennius sanguinolentus (Pallas, 1814) sampled along the Italian coast. Mar. Pollut. Bull. 122, 288-296. doi: 10.1016/j.marpolbul.2017.06.060

Ferrante, M., Signorelli, S. S., Ferlito, S. L., Grasso, A., Dimartino, A., and Copat, C. (2018a). Groundwater-based water wells characterization from guinea bissau (Western Africa): a risk evaluation for the local population. Sci. Total Environ. 619-620, 916-926. doi: 10.1016/j.scitotenv.2017.11.176

Ferrante, M., Vassallo, M., Mazzola, A., Brundo, M. V., Pecoraro, R., Grasso, A., et al. (2018b). In vivo exposure of the marine sponge Chondrilla nucula Schmidt, 1862 to cadmium $(\mathrm{Cd})$, copper $(\mathrm{Cu})$ and lead $(\mathrm{Pb})$ and its potential use for bioremediation purposes. Chemosphere 193, 1049-1057. doi: 10.1016/j. chemosphere.2017.11.144

Fossi, M., Pedà, C., Compa, M., Tsangaris, C., Alomar, C., Claro, F., et al. (2018). Bioindicators for monitoring marine litter ingestion and its impacts on mediterranean biodiversity. Environ. Pollut. 237, 1023-1040. doi: 10.1016/ j.envpol.2017.11.019

Fossi, M. C., Coppola, D., Baini, M., Giannetti, M., Guerranti, C., Marsili, L., et al. (2014). Large filter feeding marine organisms as indicators of microplastic in the pelagic environment: the case studies of the Mediterranean basking shark (Cetorhinus maximus) and fin whale (Balaenoptera physalus). Mar. Environ. Res. 100, 17-24. doi: 10.1016/j.marenvres.2014.02.002

Fossi, M. C., Panti, C., Guerranti, C., Coppola, D., Giannetti, M., Marsili, L., et al. (2012). Are baleen whales exposed to the threat of microplastics? A case study of the Mediterranean fin whale (Balaenoptera physalus). Mar. Pollut. Bull. 64, 2374-2379. doi: 10.1016/j.marpolbul.2012.08.013

Gallo, F., Fossi, C., Weber, R., Santillo, D., Sousa, J., Ingram, I., et al. (2018). Marine litter plastics and microplastics and their toxic chemicals components: the need for urgent prevetive measures. Environ. Sci. Eur. 30:13. doi: 10.1186/s12302018-0139-z 
Galloway, T. S. (2015). "Micro-and Nano-plastics and Human Health," in Marine Anthropogenic Litter, eds M. Bergmann, L. Gutow, and M. Klages (Cham: Springer).

Goldstein, M. C., and Goodwin, D. S. (2013). Gooseneck barnacles (Lepas spp.) ingest microplastic debris in the north pacific subtropical gyre. PeerJ 1:e184. doi: $10.7717 /$ peerj.184

Gore, A. C., Chappell, V. A., Fenton, S. E., Flaws, J. A., Nadal, A., Prins, G. S., et al. (2015). EDC-2: the endocrine society's second scientific statement on endocrine-disrupting chemicals. Endocr. Rev. 36, 1-150. doi: 10.1210/er.20151010

Greenwell, E. (2014). A Review of Phthalates and the Associated Reproductive and Developmental Toxicity Towards Fish. Master literature thesis - 12 EC.

Guerranti, C., Cau, A., Renzi, M., Badini, S., Grazioli, E., Perra, G., et al. (2016). Phthalates and perfluorinated alkylated substances in atlantic bluefin tuna (Thunnus thynnus) specimens from mediterranean sea (Sardinia, Italy): levels and risks for human consumption. J. Environ. Sci. Health B. 51, 661-667. doi: 10.1080/03601234.2016.1191886

Hahladakis, J. N., Velis, C. A., Weber, R., Iacovidou, E., and Purnell, P. (2018). An overview of chemical additives present in plastics: migration, release, fate and environmental impact during their use, disposal and recycling. J. Hazard Mater. 344, 179-199. doi: 10.1016/j.jhazmat.2017.10.014

Hermabessiere, L., Dehaut, A., Paul-Pont, I., Jazeque, R., Lacroix, C., Soudant, P., et al. (2017). Occurrence and effects of plastic additives on marine environments and organism: a review. Chemosphere 182, 781-793. doi: 10.1016/ j.chemosphere.2017.05.096

Jambeck, J. R., Geyer, R., Wilcox, C., Siegler, T. R., Perryman, M., Andrady, A., et al. (2015). Plastic waste inputs from land into the ocean. Science 347, 768-771. doi: $10.1126 /$ science. 1260352

Jameil, N. A. (2014). Maternal serum lead levels and risk of preeclampsia in pregnant women: a cohort study in a maternity hospital, Riyadh, Saudi Arabia. Int. J. Clin. Exp. Pathol. 7, 3182-3189.

Jones, H. B., Garside, D. A., Liu, R., and Roberts, J. C. (1993). The influence of phthalate esters on leydig cell structure and function in vitro and in vivo. Exp. Mol. Pathol. 58, 179-193. doi: 10.1006/exmp.1993.1016

Karami, A., Romano, N., Galloway, T., and Hamzah, H. (2016). Virgin microplastics cause toxicity and modulate the impacts of phenanthrene on biomarker responses in African catfish (Clarias gariepinus). Environ. Res. 151, 58-70. doi: 10.1016/j.envres.2016.07.024

Keri, R. A., Ho, S. M., Hunt, P. A., Knudsen, K. E., Soto, A. M., and Prins, G. S. (2007). An evaluation of evidence for the carcinogenic activity of bisphenol A. Reprod. Toxicol. 24, 240-252. doi: 10.1016/j.reprotox.2007. 06.008

Kluwe, W. M., Huff, J. E., Matthews, H. B., Irwin, R., and Haseman, J. K. (1985). Comparative chronic toxicities and carcinogenic potentials of 2-ethylhexylcontaining compounds in rats and mice. Carcinogenesis 6, 1577-1583. doi: 10.1093/carcin/6.11.1577

Legendre, P., and Legendre, L. (2012). Developments in environmental modelling. Num. Ecol. 24, 1-990.

Liu, T., Li, N., Zhu, J., Yu, G., Guo, K., Zhou, L., et al. (2014). Effects of di(2-ethylhexyl) phthalate on the hypothalamus-pituitary-ovarian axis in adult female rats. Reprod. Toxicol. 46, 141-147. doi: 10.1016/j.reprotox.2014.03.006

Lo Turco, V., Di Bella, G., Furci, P., Cicero, N., Pollicino, G., and Dugo, G. (2013). Heavy metals content by ICP-OES in Sarda sarda, Sardinella aurita and Lepidopus caudatus from the Strait of Messina (Sicily, Italy). Nat. Prod. Res. 27, 518-523. doi: 10.1080/14786419.2012.673611

Lusher, A. (2015). "Microplastics in the marine environment: distribution, interactions and effects," in Marine anthropogenic litter, eds M. Bergmann, L. Gutow, and M. Klages (Cham: Springer International Publishing), 245-307. doi: 10.1007/978-3-319-16510-3_10

Marine Strategy Directive [MSFD, 2008/56/EC] (2008). Directive 2008/56/EC of the European Parliament and of the Council of 17 June 2008 establishing a framework for community action in the field of marine environmental policy (Marine Strategy Framework Directive). Brussels: Commission of the European Communities.

Matozzo, V., Gagné, F., Marin, M. G., Ricciardi, F., and Blaise, C. (2008). Vitellogenin as a biomarker of exposure to estrogenic compounds in aquatic invertebrates: a review. Environ. Int. 34, 531-545. doi: 10.1016/j.envint.2007. 09.008
Moore, C. J. (2008). Synthetic polymers in the marine environment: a rapidly increasing, long-term threat. Environ. Res. 108, 131-139. doi: 10.1016/j.envres. 2008.07.025

Motawei, S. M., Attalla, S. M., Gouda, H. E., El-Harouny, M. A., and El-Mansoury, A. M. (2013). Lead level in pregnant women suffering from pre-eclampsia in Dakahlia, Egypt. Int. J. Occup. Environ. Med. 4, 36-44.

Murphy, J. (2001). Additives for Plastics Handbook. Amsterdam: Elsevier, doi: 10. 1016/B978-1-85617-370-4.X5000-3

Murray, F., and Cowie, P. R. (2011). Plastic contamination in the decapod crustacean Nephrops norvegicus Linnaeus, 1758. Mar. Pollut. Bull. 62, 12071217. doi: 10.1016/j.marpolbul.2011.03.032

Naccari, C., Cicero, N., Ferrantelli, V., Giangrosso, G., Vella, A., Macaluso, A., et al. (2015). Toxic metals in pelagic, benthic and demersal fish species from mediterranean FAO Zone 37. Bull. Environ. Contam. Toxicol. 95:567. doi: 10. 1007/s00128-015-1585-6

Paluselli, A., Fauvelle, V., Schmidt, N., Galgani, F., Net, S., and Sempéré, R. (2018). Distribution of phthalates in marseille bay (NW Mediterranean Sea). Sci. Total. Environ. 621, 578-587. doi: 10.1016/j.scitotenv.2017.11.306

Pappalardo, A. M., Copat, C., Ferrito, V., Grasso, A., and Ferrante, M. (2017). Heavy metal content and molecular species identification in canned tuna: insights into human food safety. Mol. Med. Rep. 15, 3430-3437. doi: 10.3892/ mmr.2017.6376

Pecoraro, R., D’Angelo, D., Filice, S., Scalese, S., Capparucci, F., Marino, F., et al. (2018). Toxicity evaluation of graphene oxide and titania loaded nafion membranes in zebrafish. Front. Physiol. 8:1039. doi: 10.3389/fphys.2017.01039

Piazzolla, D., Scanu, S., Manfredi Frattarelli, F., Mancini, E., Tiralongo, F., Brundo, M. V., et al. (2015). Trace-metal enrichment and pollution in coastal sediments in the northern tyrrhenian sea. Italy. Arch. Environ. Contam. Toxicol. 69, 470-481. doi: 10.1007/s00244-015-0166-3

Powell, J. J., Faria, N., Thomas-McKay, E., and Pele, L. C. (2010). Origin and fate of dietary nanoparticles and microparticles in the gastrointestinal tract. J. Autoimmun. 34, 226-233. doi: 10.1016/j.jaut.2009.11.006

Quansah, R., Armah, F. A., Essumang, D. K., Luginaah, I., Clarke, E., Marfoh, K., et al. (2015). Association of arsenic with adverse pregnancy outcomes/infant mortality: a systematic review and meta-analysis. Environ. Health Perspect. 123, 412-421. doi: 10.1289/ehp.1307894

Richter, C. A., Birnbaum, L. S., Farabollini, F., Newbold, R. R., Rubin, B. S., Talsness, C. E., et al. (2007). In vivo effects of bisphenol A in laboratory rodent studies. Reprod. Toxicol. 24, 199-224. doi: 10.1016/j.reprotox.2007. 06.004

Rios, L. M., Moore, C., and Jones, P. R. (2007). Persistent organic pollutants carried by synthetic polymers in the ocean environment. Mar. Poll. Bull. 54, 1230-1237. doi: 10.1016/j.marpolbul.2007.03.022

Rochman, C. M., Hentschel, B. T., and Teh, S. J. (2014). Long-term sorption of metals is similar among plastic types: implications for plastic debris in aquatic environments. PLoS One 9:e85433. doi: 10.1371/journal.pone.00 85433

Romeo, T., Peda, C., Fossi, M. C., Andaloro, F., and Battaglia, P. (2016). First record of plastic debris in the stomach of mediterranean lantern fishes. Acta Adriat. 57, 115-124.

Sackett, D. K., Cope, W. G., Rice, J. A., and Aday, D. D. (2013). The influence of fish length on tissue mercury dynamics: implications for natural resource management and human health risk. Int. J. Environ. Res. Public Health 10, 638-659. doi: 10.3390/ijerph10020638

Salvaggio, A., Pecoraro, R., Scalisi, E. M., Tibullo, D., Lombardo, B. M., Messina, G., et al. (2017). Morphostructural and immunohistochemical study on the role of metallothionein in the detoxification of heavy metals in Apis mellifera L., 1758. Microsc. Res. Tech. 80, 1215-1220. doi: 10.1002/jemt.22919

Savoca, D., Arculeo, M., Barreca, S., Buscemi, S., Caracappa, S., Gentile, A., et al. (2018). Chasing phthalates in tissue of marine turtles from the Mediterranean Sea. Mar. Pollut. Bull. 127, 165-169. doi: 10.1016/j.marpolbul.2017.11.069

Scanu, S., Piazzolla, D., Manfredi Frattarelli, F., Mancini, E., Tiralongo, F., Brundo, M. V., et al. (2016). Mercury enrichment in sediments of the coastal area of northern Latium, Italy. Bull. Environ. Contam. Toxicol. 96, 630-637. doi: 10.1007/s00128-016-1776-9

Seltenrich, N. (2015). New link in the food chain? Marine plastic pollution and seafood safety. Environ. Health Perspect. 123, A34-A41. doi: 10.1289/ehp. 123-A34 
Sindermann, C. J. (1995). "Adaptation and survival or disability and death in polluted habitats," in Ocean Pollution: Effects on Living Resources and Humans, eds M. J. Kennish and P. L. Lutz (Boca Raton, CA: CRC Press), 19-32.

Soto, A. M., Brisken, C., Schaeberle, C., and Sonnenschein, C. (2013). Does cancer start in the womb? Altered mammary gland development and predisposition to breast cancer due to in utero exposure to endocrine disruptors. J. Mammary Gland Biol. Neoplasia 18, 199-208. doi: 10.1007/s10911-0139293-5

Stojanoska, M. M., Milosevic, N., Milic, N., and Abenavoli, L. (2017). The influence of phthalates and bisphenol A on the obesity development and glucose metabolism disorders. Endocrine 55, 666-681. doi: 10.1007/s12020-0161158-4

Storelli, M. M., Barone, G., Piscitelli, G., and Marcotrigiano, G. O. (2007). Mercury in fish: concentration vs. fish size and estimates of mercury intake. Food Addit. Contam. 24, 1353-1357. doi: 10.1080/026520307013 87197

Teuten, E. L., Saquing, J. M., Knappe, D. R., Barlaz, M. A., Jonsson, S., Björn, A., et al. (2009). Transport and release of chemicals from plastics to the environment and to wildlife. Philos. Trans. R. Soc. Lond. B Biol. Sci. 364, 2027-2045. doi: 10.1098/rstb.2008.0284

Turchetti, B., Goretti, M., Branda, E., Diolaiuti, G., D’Agata, C., Smiraglia, C., et al. (2013). Influence of abiotic variables on culturable yeast diversity in two distinct Alpine glaciers. FEMS Microbiol. Ecol. 86, 327-340. doi: 10.1111/1574-6941. 12164

UNEP (2016). Marine Plastic Debris and Microplastics. Global Lessons and Research to Inspire Action and Guide Policy Change. Nairobi: United Nations Environment Programme.

Valton, A. S., Serre-Dargnat, C., Blanchard, M., Alliot, F., Chevreuil, M., and Teil, M. J. (2014). Determination of phthalates and their byproducts in tissues of roach (Rutilus rutilus) from the Orge river (France). Environ. Sci. Pollut. Res. Int. 21, 12723-12730. doi: 10.1007/s11356-0143213-0

Viarengo, A., Burlando, B., Ceratto, N., and Panfoli, I. (2000). Antioxidant role of metallothioneins: a comparative overview. Cell Mol. Biol. 46, 407-417.
Viarengo, A., Burlando, B., Dondero, F., Marro, A., and Fabbri, R. (1999). Metallothionein as a tool in biomonitoring programmes. Biomarkers 4, 455466. doi: 10.1080/135475099230615

Watts, A., Lewis, C., Goodhead, R. M., Beckett, D. J., Moger, J., Tyler, C., et al. (2014). Uptake and retention of microplastics by the shore crab Carcinus maenas. Environ. Sci. Technol. 48, 8823-8830. doi: 10.1021/es501090e

Wright, S. L., and Kelly, F. J. (2017). Plastic and human health: a micro issue? Environ. Sci. Technol. 51, 6634-6647. doi: 10.1021/acs.est.7b00423

Wright, S. L., Thompson, R. C., and Galloway, T. S. (2013). The physical impacts of micro-plastics on marine organisms: a review. Environ. Pollut. 178, 483-492. doi: 10.1016/j.envpol.2013.02.031

Zuccarello, P., Ferrante, M., Cristaldi, A., Copat, C., Grasso, A., Sangregorio, D., et al. (2019). Exposure to microplastics $(<10 \mu \mathrm{m})$ associated to plastic bottles mineral water consumption: the first quantitative study. Water Res. 157, 365-371. doi: 10.1016/j.watres.2019.03.091

Zuccarello, P., Oliveri Conti, G., Cavallaro, F., Copat, C., Cristaldi, A., Fiore, M., et al. (2018). Implication of dietary phthalates in breast cancer. A systematic review. Food Chem. Toxicol. 118, 667-674. doi: 10.1016/j.fct.2018. 06.011

Conflict of Interest Statement: The authors declare that the research was conducted in the absence of any commercial or financial relationships that could be construed as a potential conflict of interest.

The reviewer $\mathrm{AZ}$ and handling Editor declared their shared affiliation at the time of review.

Copyright (c) 2019 Salvaggio, Tiralongo, Krasakopoulou, Marmara, Giovos, Crupi, Messina, Lombardo, Marzullo, Pecoraro, Scalisi, Copat, Zuccarello, Ferrante and Brundo. This is an open-access article distributed under the terms of the Creative Commons Attribution License (CC BY). The use, distribution or reproduction in other forums is permitted, provided the original author(s) and the copyright owner(s) are credited and that the original publication in this journal is cited, in accordance with accepted academic practice. No use, distribution or reproduction is permitted which does not comply with these terms. 